\title{
The GGOS as the backbone for global observing and local monitoring: A user driven perspective
}

\author{
H.-P. Plag* \\ Geodetic Institute, Norwegian Mapping Authority, Honefoss, Norway
}

Received 1 September 2003; accepted 21 June 2005

\begin{abstract}
A key geodetic contribution to both the three Global Observing Systems and initiatives like the European Global Monitoring for Environment and Security is an accurate, long-term stable, and easily accessible reference frame as the backbone. Many emerging scientific as well as non-scientific high-accuracy applications require access to an unique, technique-independent reference frame decontaminated for short-term fluctuations due to global Earth system processes. Such a reference frame can only be maintained and made available through an observing system such as the Global Geodetic Observing System (GGOS), which is currently implemented and expected to provide sufficient information on changes in the Earth figure, its rotation and its gravity field. Based on a number of examples from monitoring of infrastructure, point positioning, maintenance of national references frames to global changes studies, likely future accuracy requirements for a global terrestrial reference frame are set up as function of time scales. Expected accuracy requirements for a large range of high-accuracy applications are less than $5 \mathrm{~mm}$ for diurnal and sub-diurnal time scales, $2-3 \mathrm{~mm}$ on monthly to seasonal time scales, better than $1 \mathrm{~mm} /$ year on decadal to 50 years time scales. Based on these requirements, specifications for a geodetic observing system meeting the accuracy requirements can be derived.
\end{abstract}

(c) 2005 Elsevier Ltd. All rights reserved.

Keywords: Terrestrial reference frame; Global Geodetic Observing System; Global monitoring; User requirements

\section{Introduction}

Comprehensive monitoring of the Earth system is a prerequisite for understanding the Earth system processes and thus, ultimately for achieving a sustainable development. During the last two decades, the need for a global monitoring of the Earth has been recognised in particular by the United Nations in setting up the Earth Watch programme and initiating Global Observing Systems.

The space-geodetic techniques are apt to provide both the reference frame for Earth system observations as well as observations of crucial parameters related to changes in the Earth's geometry, rotation and gravity field. However, Plag (2000) pointed out that none of the emerging observing systems includes a geodetic component nor is it directly connected to the extensive global geodetic observing networks established over the last decade. Thus, the fundamental role of geodesy as the backbone for all Earth observations is not formally accounted for in the present systems. It

\footnotetext{
* Present address: Nevada Bureau of Mines and Geology, University of Nevada, Reno, Mailstop 178, Reno, NV 89557, USA.

E-mail address: hpplag@unr.edu.
} 
is, however, expected that the current discussion in the frame of the Group on Earth Observations $\left(\mathrm{GEO}^{1}\right)$ will lead to a broad acknowledgement of the fundamental importance of a sufficiently accurate terrestrial reference frame and geodetic observations for Earth observations.

Increasingly, access to highly accurate geodetic positions is demanded for many scientific and non-scientific applications. This is equivalent to requiring access to an unique, technique-independent reference frame decontaminated for short-term fluctations due to global Earth system processes. Providing instantaneous and ad hoc access to highly accurate positions in such a unique, global, long-term stable reference frame would considerably ease present applications and open up for many new applications, particularly if combined with the rapidly developing communication tools and geo-databases.

Techniques like GPS and the future Galileo are, in principle, able to provide such positions relative to a unique, global reference frame ad hoc, i.e. without simultaneous measurements at local reference points. However, only the combination of the space-geodetic techniques into an integrated system monitoring the Earth surface kinematic, rotational pertubations and gravity field changes will eventually allow to realise the reference frame with sufficient accuracy and stability.

Responding to the emerging scientific and practical requirements with respect to geodesy, the International Association of Geodesy (IAG) has over the last 5 years developed the idea of an Global Geodetic Observing System (GGOS, originally named Integrated Global Geodetic Observing System, IGGOS, see e.g. Rummel et al., 2002). The combination and integration of the geodetic observation techniques into such a system is a mandatory step in order to exploit the full potential of the techniques and to provide the backbone for an Earth monitoring system as well as to meet the requirements of most of the emerging applications.

The IGGOS as proposed by Rummel et al. (2002) and further specified by Beutler et al. (2003) "aims at maintaining the stability of and providing the ready access to the existing time series of geometric and gravimetric reference frames by ensuring the generation of uninterrupted time series of state-of-the-art global observations related to the three pillars of geodesy". The three pillars of geodesy are considered to be (1) geometry and kinematics, (2) Earth orientation and rotation, and (3) gravity field and its variability.

In designing the GGOS, it may be worthwhile to take, at least for a moment, a user-driven top-down approach. Here, we will discuss requirements and consequences for the implementation of the GGOS both with respect to Earth system monitoring and ad hoc determination of geodetic positions. In doing so, we will focus on the reference frame issue. Starting from the Integrated Global Observing Strategy (IGOS), the contribution of the GGOS to the three Global Observing Systems (G3OS) is discussed (Section 2). Different examples of ad hoc positioning, which is introduced in Section 3, are used in Section 4 to condense a set of future user requirements (UR) for access to geodetic positions in terms of accuracy and latency. Finally, consequences for the goals and implementation of the GGOS are ventilated (Section 5).

\section{Global monitoring of the Earth system}

Recognising the need for global monitoring as a prerequiste for understanding the impact of mankind on the Earth system and to devise actions to mitigate the predicted changes particularly in the climate system, three Global Observing Systems for Climate (GCOS), Terrestrial (GTOS) and Ocean (GOOS) were initiated and partly established under the United Nations Environmental Programme's (UNEP) Earth Watch activities ${ }^{2}$. These systems are developing rapidly following the $\operatorname{IGOS}^{3}$.

The IGOS emphasises the necessary transition from a research state to operational observing systems (see e.g., Williams and Townshend, 1998). This is justified by the requirements for Earth system observations resulting from the nature of the processes in the Earth system. Key requirements for sustainable monitoring which is the overall goal of the observing systems are that it is long-term and homogeneous in time. These requirements can only be met by operational observation systems. Moreover, IGOS requests the monitoring to be multi-parameter, global and integrated. Another focus of IGOS is on the data archives, as well as the accessibility of data, products and information for users. Here, it is

\footnotetext{
1 see http://earthobservations.org.

2 See http://earthwatch.unep.net.

${ }^{3}$ See http://www.igospartners.org and particularly the IGOS umbrella document available there.
} 
Intergovernmental Panel of

Global Monitoring (IPGM)

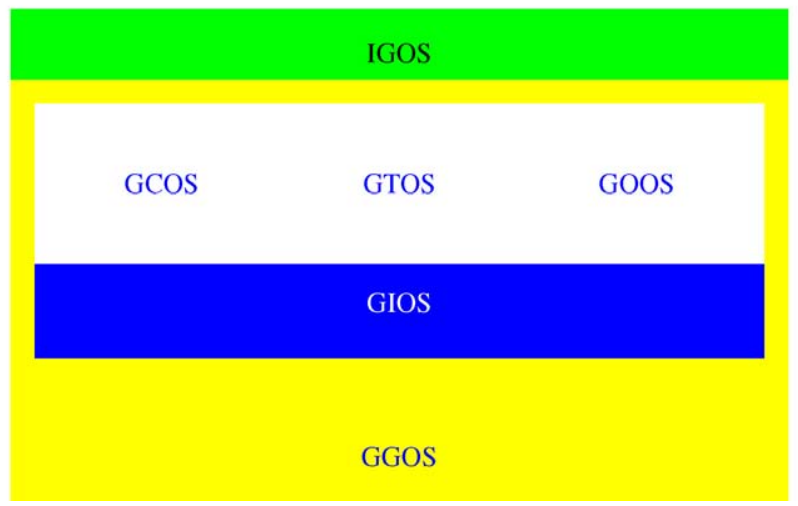

Fig. 1. Towards a comprehensive monitoring system. A comprehensive monitoring system would collect all the different networks under a common integrated observing strategy, i.e. IGOS. A solid basis of in situ data for the three emerging Global Observing Systems GCOS (climate), GTOS (terrestrial) and GOOS (ocean), which are still strongly focusing on remote sensing, could be provided by a Ground-based In-situ Observing Systems (GIOS), which ideally would integrate as far as possible most of the ground-based observational sites. Moreover, the GIOS would deliver an integrated set of observations of geophysical quantities related, e.g. to the seismicity of the Earth and the Earth magnetic field. As a part of GIOS, the GGOS would contribute important observations related to the geometry, potential field and dynamics of the Earth. At the same time and equally important, the GGOS would be a utility for the other components, providing the reference frame. Plag (2000) suggested that progress in the development of the monitoring should be assessed at a high level, preferably through an Intergovernmental Panel on Global Monitoring (IPGM) comparable to the Intergovernmental Panel on Climate Change (IPCC). The Figure is adopted from Plag (2000).

stated that data archives resulting from Earth observing systems must be integrated, quality-controlled, homogeneous, consistent, and last but not least accessible. The last point cannot be overemphasised and currently, research in many areas is hampered by difficulties in accessing relevant databases.

A key geodetic contribution to both the G3OS and initiatives like the European Global Monitoring for Environment and Security (GMES) is an accurate, long-term stable, and easily accessible reference frame as the backbone for all observations. At the same time, geodetic observation techniques provide data related to the geometry, gravity field, and dynamics of the Earth. Fig. 1 sketches a scenario where the emerging G3OS, complemented by an integrated ground-based in situ component form an Earth monitoring system based on a global geodetic observing systems as a backbone.

\section{Ad hoc positioning and the reference frame}

Traditionally, highly accurate geodetic positions were determined through measurements relative to a set of local reference points with known (fixed) coordinates in a local or regional reference system. The advent of the space geodetic techniques allows for the first time the realisation of a global reference system through a globally homogeneous reference frame. Moreover, techniques like GPS and the future Galileo allow to determine positions relative to the global reference frame ad hoc (i.e. independent of simultaneous measurements at local reference points) with increasing accuracy and decreasing latency.

In the so-called precise point positioning (PPP) method (Zumberge et al., 1997), access to the reference frame is given through highly accurate satellite orbits and clocks, which in turn are determined on the basis and relative to a global network of tracking stations. However, a reference frame sufficiently accurate and stable in time to satisfy most user requirements can only be maintained and made available through an integrated global geodetic observing system providing highly accurate and comprehensive information on changes in the Earth's figure, rotation and gravity field. A likely candidate for this future unique global reference frame, in fact, is the realisation of the International Terrestrial Reference System (ITRS) through the International Terrestrial Reference Frame (ITRF). This notion is also supported by the fact that the European Union and the USA recently agreed to align the reference frames of GPS and the future Galileo system as closely as possible to ITRS (European Commission, 2004). 

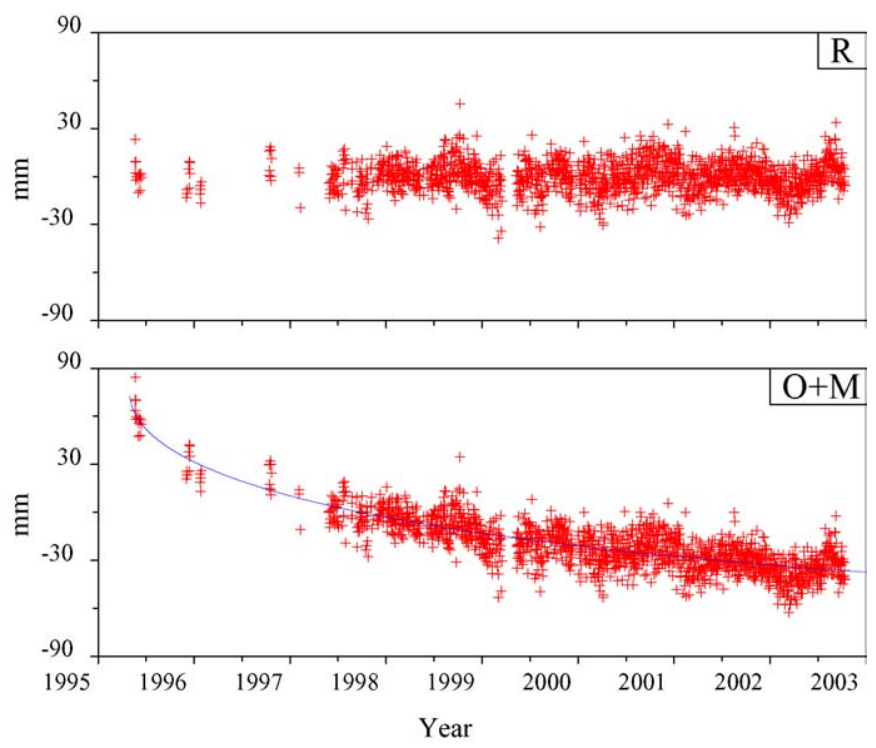

Fig. 2. Monitoring of vertical motion. Lower diagram: Daily vertical coordinates (symbols) determined from GPS measurements on an oil platform and fitted settlement curve ( $\sqrt{t}$ plus linear trend, where $t$ is time, solid line). Upper diagram: residuals with respect to the settlement curve.

Based on a number of examples from monitoring of infrastructure, point positioning, maintenance of national references frames to global changes studies, likely future accuracy requirements for a global terrestrial reference frame as well as access to it can be set up as function of time scales.

\section{User requirements}

GPS on its own does not satisfy the requirements of applications requiring high-accuracy and/or long-term stability. One crucial initiative to improve the accuracy of GPS has been the International GPS Service (IGS), which initially served the needs of many scientific applications. The excellent service provided by IGS to scientific and increasingly non-scientific communities is possible through a global network of GPS tracking stations, which currently comprises around 300 stations. Based on this network and a coordinated analysis effort, rapid and precise orbits and satellite clocks are provided together with ionospheric models and tropospheric products. These products meet many though not all requirements of high-accuracy applications allowing for considerable latency. Experience with applications of IGS products over the last 10 years forms an excellent basis to assess future user requirements.

In monitoring different aspects of the Earth system, such as motion of the tectonic plates, pre-, co- and post-seismic strain, measuring changes in sea level and ice covers, variations of the Earth gravity field, GPS augmented with the products of the IGS (denoted here as GPS\&IGS) has served both as the primary measuring device (e.g. for observing plate kinematics, seismic strain, and vertical land motion required to detect absolute sea level changes) as well as a tool to position sensors with unprecedented accuracy (e.g. in airborne gravimetry, hydrographic survey, satellite altimetry). Ten years of experience including comparison to model predictions have shown that requirements are of the order of better than $1 \mathrm{~cm}$ in daily or sub-daily positions and better than $1 \mathrm{~mm} /$ year in secular stability.

Increasingly, GPS\&IGS is used to monitor the motion and stability of large infrastructures such as oil platforms, reservoir dams and bridges. In some cases, these measurements can be carried out relative to a reference point that can be assumed to be stable. However, in many cases no such point can be identified unanimously and the optimal reference would be a regional or global network. Our experience with oil platforms shows that user requirements for monitoring of such infrastructure are of the order of less than $1 \mathrm{~cm}$ for sub-daily positions available with a latency of a few days and $1 \mathrm{~mm} /$ year for long-term stability. Similar requirements apply to reservoir dams and large bridge; however, here the tolerable latency may be much lower.

Fig. 2 gives an example of monitoring the motion of an oil platform with the help of GPS. The vertical movement of the platform is dominated by the settlement into the ocean floor, which is theoretically expected to be proportional to $\sqrt{t}$, where $t$ is time. Daily coordinates determined from GPS measurements with PPP allow to estimate physically 


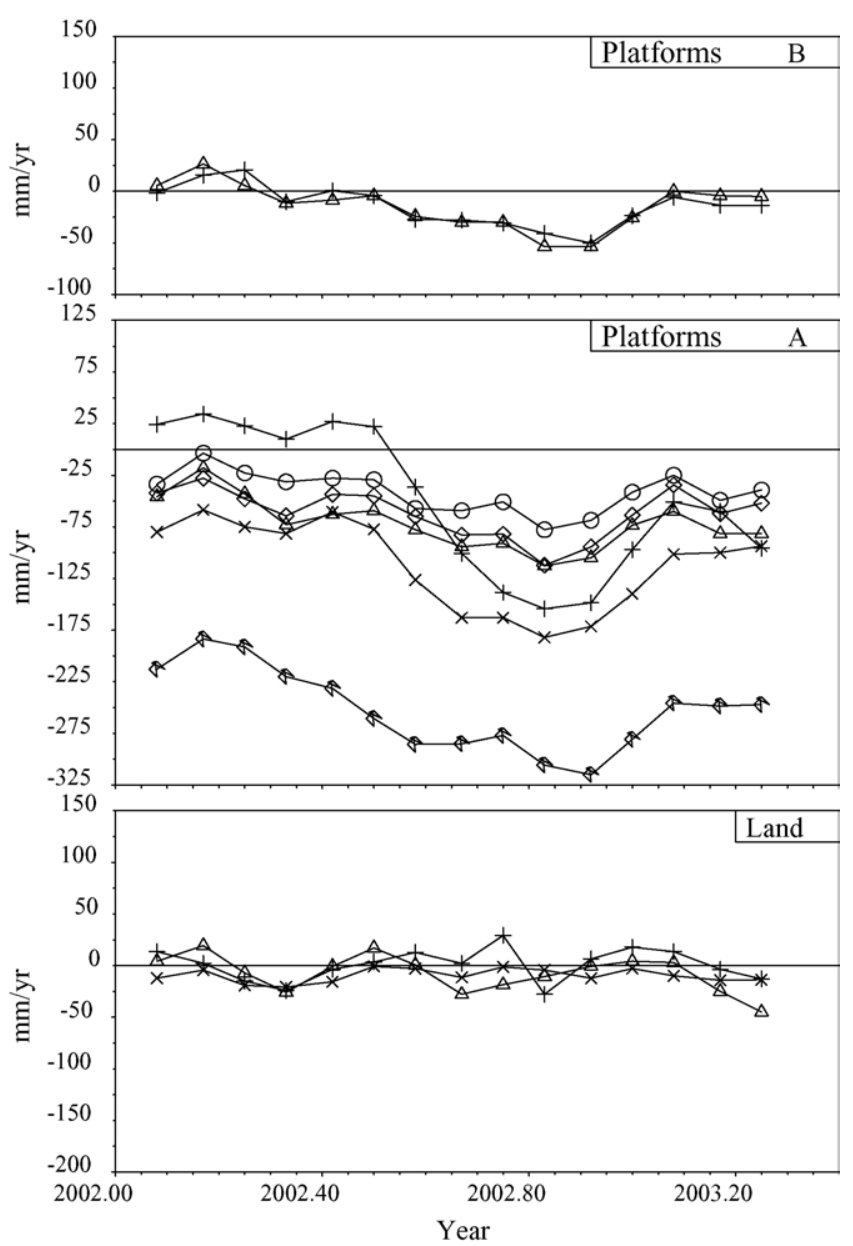

Fig. 3. Monitoring of vertical velocities. Vertical velocities determined on the basis of a moving 4-month window. Upper diagram: For two offshore platforms assumed to be not or very moderately subsiding. Middle diagram: For six different off-shore installations known to be subsiding and separated by up to $30 \mathrm{~km}$. Lower diagram: For three reference stations in Europe (namely Stavanger, Norway, Herstmonceux, UK and Westerbork, The Netherlands). The velocities are computed from time series of daily coordinates determined by PPP. No corrections for CMEs have been made. The velocities of the reference stations on land indicate the order of magnitude of CME effects on velocities.

relevant parameters of the settlement curve. Combining the GPS observations with independent measurements of the settlement, which are obtained by monitoring the movement of the platform with respect to an invar rod solidly fixed to the ocean bottom, allow to determine the motion of the sea floor in a geocentric reference frame. This, in turn, is important information for reservoir modelling.

In the example, sea floor turns out to be uplifting by a few mm/year relative to ITRF2000, which is the latest realisation of ITRS (Altamimi et al., 2002). A key question is how much this number is affected by long-term trends in ITRF2000 and its approximation through GPS. The residual vertical motion with respect to the settlement curve contain intra-annual variations of the order of $\pm 2 \mathrm{~cm}$, which are largely due to so-called Common Mode Errors (CME) affecting PPP based on GPS.

Another example is the determination of instantaneous subsidence rates of oil platforms on monthly to annual time scales. In the absence of a local stable reference frame, the global network of IGS tracking stations can be used as reference. Time series of daily coordinates determined by PPP allow to determine velocities on the basis of a moving window. In Fig. 3, the vertical velocities resulting from a 4-month moving window are shown for both off-shore locations (oil platforms) and reference stations on land. The reference stations (selected arbitrarily in the region around the North Sea) are Stavanger in Norway, Herstmonceux in the UK, and Westerbork in the Netherlands. The sites, though separated by up to thousand $\mathrm{km}$, show some coherent variations, which indicate the effect of CME with large 
spatial scales and temporal scales of several months on the velocities. The velocities for the platforms show larger variations than those found at the reference sites. However, decontamination of the coordinate time series for the CMEs prior to the determination of velocities is a crucial issue. For that, the global network of tracking stations provides the observational basis.

Highly accurate positioning of sensors, for e.g. airborne gravimetry and hydrographic surveys require on the one hand positions with high temporal resolution (down to $1 \mathrm{~s}$ ) and accuracy of the order of $10 \mathrm{~cm}$. On the other hand, they also require a high long-term stability as measurements are carried out over long time intervals (decades) and should be interconnectable without loss of accuracy. Hydrographic surveys on, for e.g. oilfields require an accuracy of $5 \mathrm{~cm}$ over a time span of up to 50 years, which is equivalent to a long-term stability of $1 \mathrm{~mm} /$ year.

Here, it should be mentioned that the rate at which geo-databases are collected today has increased by several orders of magnitude over the last few decades. The database collected today can be expected to be in use over many years to come. Even without assuming likely increased future requirements for the accuracy this will demand a high long-term stability of the reference frame used for the databases.

GPS is increasingly used for control of processes in, e.g. agriculture, construction work and maintenance. For all these applications, a high-accuracy of $10 \mathrm{~cm}$ (for most agricultural applications) down to $1 \mathrm{~cm}$ (for snow clearing) and even sub-centimetre (for construction work) is required in real time. Currently, for all these applications, local augmentation systems have to be set up.

For the most demanding land surveying tasks such as determination of real estate boundaries in densely populated areas (with high values of real estate) or mapping of underground cables and pipelines in cities, accuracy requirements are of the order of $5 \mathrm{~cm}$ with low latency. The cost of surveys strongly depends on the time needed to achieve this accuracy and the integrity and availability of the system. Having access to a reliable position in near-real time would greatly ease the surveying tasks and reduce the costs.

For most surveying tasks, it is crucial to be able to relate (time dependent) coordinates given in the global reference frame to (time fixed) coordinates in the national reference frame. In order to transform ad hoc coordinates in ITRF to national coordinates, a detailed knowledge of the velocity field of the Earth surface with accuracy better than $1 \mathrm{~mm} /$ year is required. An error of $1 \mathrm{~mm} /$ year introduces already an error of $1 \mathrm{~cm}$ in ad hoc positions over 10 years. In some regions, plate tectonic models provide a first order approximation to the horizontal velocity field. However, in many regions intra-plate deformations exceeding the $1 \mathrm{~mm} /$ year level require more detailed (empirical) models. For the height component, even first order models are missing, except for post-glacial rebound in and around the formerly glaciated areas.

When travelling through the troposphere, the electromagnetic waves experience a path delay, which can be used to extract information on the precipitable water content of the troposphere. Currently, the meteorological observation systems do not provide sufficient information on this valuable parameter. In the context of the COST Action $716^{4}$, it is demonstrated that having available the path delays in near-real time (1-2 $\mathrm{h}$ latency) is an asset for numerical weather prediction, and a future application of ground-based GPS or Galileo network for numerical weather prediction and climate seems likely. The quality of the path delays depends on the orbit and clock accuracy and the accuracy in position for the sensor stations. The accuracy requirements for numerical weather prediction applications are of the order of $1 \mathrm{~cm}$ in day-to-day position while climate applications require a long-term stability of the order of $1 \mathrm{~mm} / \mathrm{year}$ or better.

\section{Conclusions}

In summary, we can identify three main user categories (UC) for high-accuracy applications requiring or benefiting from ad hoc positioning. These categories are defined on the basis of the allowable latency. "Real time positioning" constitutes the first category (UC1). For these users, the most extreme accuracy requirements are expected to be considerably lower than $10 \mathrm{~cm}$ and in some cases even below $1 \mathrm{~cm}$. Some real time applications will require high integrity (e.g. process control) and high update rates. The next category (UC2) comprises "Near-real time positioning and other near-real time applications". Here, accuracy requirements will be close to $1 \mathrm{~cm}$ in most of these applications (monitoring of infrastructure, meteorological applications) while other applications will

\footnotetext{
${ }^{4}$ See e.g. http://www.oso.chalmers.se/kge/cost716.html.
} 
require less accuracy (e.g. of the order of $5 \mathrm{~cm}$ ) but higher integrity (e.g. land surveying). Finally, UC3 includes all "Post-processing with extreme requirements". Most of these applications can accept considerable latency but will require accuracy at the $1 \mathrm{~cm}$ level for daily coordinates and a few millimetres on intra-annual time scales. For long-term monitoring tasks, $1 \mathrm{~mm} /$ year in stability seems to be a critical boundary both for scientific and non-scientific tasks. This number also applies to collection of geo-databases, which are to be maintained over time scales of several decades.

Depending on the time scale, we see the following latency and accuracy requirements for high-accuracy applications:

- UR1: better than $10 \mathrm{~cm}$ in real time and near-real time;

- UR2: less than $5 \mathrm{~mm}$ for diurnal and sub-diurnal time scales, latency hours to days;

- UR3: 2-3 mm on monthly to seasonal time scales;

- UR4: better than $1 \mathrm{~mm} /$ year on decadal to 50 years time scales.

Presently, GPS\&IGS satisfies most of the requirements for UC3, though the stability of this combined system is still not meeting the $1 \mathrm{~mm} /$ year limit due to deficiencies in the stability of the underlying ITRF. Moreover, too many and uncoordinated changes in the IGS tracking network with respect to number of stations, hardware, software, processing strategy, and theory for programs further decrease the stability of the system. Thus, the GPS\&IGS system still appears to be in a research and pre-operational state.

GPS\&IGS does not meet the UC1 requirements due to properties of the GPS-alone system combined with the large latency for crucial IGS products. For this user category, local and regional augmentations are currently required.

Some but not all needs of the UC2 are met by GPS\&IGS but either the large latency of the precise IGS products or the insufficient accuracy of the rapid IGS products leave a considerable share of this user category in the need of local or regional augmentation systems.

While UR1 and partly UR2 can be met by local to wide-area augmentation systems there are UR3 and UR4 requirements that depend crucially on the quality of ITRF and the available products. Moreover, achieving UR1 and UR2 through a Signal-in-Space Only system would considerable increase the areas of applications and provide significant economic advantages.

In order to meet the user requirements and to serve the needs of the user categories specified above, the following general system specifications could be set up for the GGOS and the ITRF:

\section{- Contribution to G3OS:}

- long-term operation for the reference frame as well as the contribution of geodetic observations to G3OS (long observational records);

- operational kernel, providing long-term stability;

- integrated, homogeneous (in time), and accessible databases.

- Access to the ITRF:

- products given in "single-technique independent" ITRF;

$\circ$ provision of detailed information about system state, including technique dependent effects (CME) and the Earth's surface velocity field;

- quality-assured and reliable products;

- progress towards an "easy", operational access to ad hoc positions in ITRF.

Finally, it is emphasised here that in order to serve both scientific and non-scientific users for high-accuracy applications, it is mandatory that the GGOS include a sufficient operational core system, which is as stable as possible over a long time. It is this specification which will be most difficult to meet considering both the expected progress in technology and changes in funding sources and priorities as well as the key players and their interests.

\section{Acknowledgement}

The ideas presented here are the result of many discussions with my colleagues at the Geodetic Institute of the Norwegian Mapping Authority (NMA) and collaborators in international projects, particularly the "Geodetic Galileo" 
project funded by the European Space Agency in the context of the GALILEO System Testbed Version 1. For the latter, I specifically mention Jan Johansson, Onsala Space Observatory. The data processing for Figs. 2 and 3 was carried out by Oddgeir Kristiansen and Halfdan Pascal Kierulf, both NMA, respectively. I would like to thank all colleagues, also those not mentioned here explicitely, who have contributed to the development of the ideas described above. I also would like to thank two anonymous referees for helpful comments and corrections.

\section{References}

Altamimi, Z., Sillard, P., Boucher, C., 2002. A new release of the International Terrestrial Reference Frame for earth science applications. J. Geophys. Res., 2214, 10.1029/2001JB000561.

Beutler, G., Drewes, H., Reigber, C., Rummel, R., 2003. Proposal to Establish the Integrated Global Geodetic Observing System (IGGOS) as IAG's First Project, distributed to IVS and IGS mailing lists on 21 August 2003.

European Commission, 2004. Agreement on the promotion, provision and use of galileo and GPS satellite-based navigation systems and related applications, Available at http://europe.eu.int/comm/dgs/energy_tansport-/galileo/documents\%-/doc/2004_06_21_summit_2004_en.pdf.

Plag, H.-P., 2000. Integration of geodetic techniques into a global Earth monitoring system and its implication for Earth system sciences, Towards an Integrated Global Geodetic Observing System. In: Rum-mel, R., Drewes, H., Bosch, W., Hornik, H., (Eds.), vol. 120 of International Association of Geodesy Symposia, Springer, Berlin, pp. 73-83.

Rummel, R., Drewes, H., Beutler, G., 2002. Integrated Global Geodetic Observing System (IGGOS): a candidate IAG project. In: Vistas for Geodesy in the New Millennium, Adam, J., Schwarx, K.-P. (Eds.), vol. 125 of International Association of Geodesy Symposia, Springer, Berlin, pp. 609-614.

Williams, D., Townshend, J.R.G., 1998. The concept of an Integrated Global Observing Strategy. In: Proceedings for the 27th International Symposium on Remote Sensing of Environment: Information for Sustainability, June 8-12, Troms $\phi$, Norway, Norwegian Space Centre, pp. 95-98.

Zumberge, J.F, Heflin, M.B., Jefferson, D.C., Watkins, M.M., 1997. Precise point positioning for the efficient and robust analysis of GPS data from large networks. J. Geophys. Res. 102, 5005-5017. 